\title{
Score for the Survival Probability in Metastasis Breast Cancer: A Nomogram-Based Risk Assessment Model
}

\author{
Zhenchong Xiong, MD',2,3 \\ Guangzheng Deng, MD ${ }^{1,23}$ \\ Xinjian Huang, MD ${ }^{1,2,3}$ \\ Xing Li, $\mathrm{PhD}^{1,23}$ \\ Xinhua Xie, $\mathrm{PhD}^{1,23}$ \\ Jin Wang, $P h D^{1,2,3}$ \\ Zeyu Shuang, $P h D^{1,23}$ \\ Xi Wang, $\mathrm{PhD}^{1,2,3}$
}

\begin{abstract}
Purpose
Survival of metastatic breast cancer (MBC) patient remains unknown and varies greatly from person to person. Thus, we aimed to construct a nomogram to quantify the survival probability of patients with MBC.
\end{abstract}

\begin{abstract}
Materials and Methods
We had included 793 MBC patients and calculated trends of case fatality rate by KaplanMeier method and joinpoint regression. Six hundred thirty-four patients with MBC between January 2004 and July 2011 and 159 patients with MBC between August 2011 and July 2013 were assigned to training cohort and internal validation cohort, respectively. We constructed the nomogram based on the results of univariable and multivariable Cox regression analyses in the training cohort and validated the nomogram in the validation cohort. Concordance index and calibration curves were used to assess the effectiveness of nomogram.
\end{abstract}

\section{Results}

Case fatality rate of MBC was increasing (annual percentage change [APC], 21.6; 95\% confidence interval $[\mathrm{Cl}], 1.0$ to $46.3 ; \mathrm{p}<0.05$ ) in the first 18 months and then decreased (APC, $-4.5 ; 95 \% \mathrm{Cl},-8.2$ to $-0.7 ; \mathrm{p}<0.05)$. Metastasis-free interval, age, metastasis location, and hormone receptor status were independent prognostic factors and were included in the nomogram, which had a concordance index of 0.69 in the training cohort and 0.67 in the validation cohort. Calibration curves indicated good consistency between the two cohorts at 1 and 3 years.

\section{Conclusion}

In conclusion, the fatality risk of MBC was increasing and reached the summit between 13th and 18th month after the detection of MBC. We have developed and validated a nomogram to predict the 1-and 3-year survival probability in MBC.

\author{
Sun Yat-sen University Cancer Center, \\ 651 Dongfeng Road East, \\ Guangzhou 510060, China \\ Tel: 86-13609648985 \\ Fax: 86-87343852 \\ E-mail: wangxi@sysucc.org.cn
}

Received September 18, 2017

Accepted December 28, 2017

Published Online January 2, 2018

*Zhenchong Xiong, Guangzheng Deng, and

Xinjian Huang contributed equally to this work.
Key words

Breast neoplasms, Metastasis, Mortality rate, Survival, Nomogram

\section{Introduction}

Metastatic breast cancer (MBC) is diagnosed in nearly 30\% of breast cancer patients, and is the major cause of cancerrelated deaths among breast cancer patients [1]. In most patients with $\mathrm{MBC}$, the median survival ranges from 2 to 3 years [2,3]. Various therapeutic approaches have been used in $\mathrm{MBC}$, and most of physicians consider the information of survival as the most critical basis for therapy selection in
MBC [4,5]. However, the outcomes of MBC cannot be predicted in the large majority of patients, as most prognosis models were developed for early-stage breast cancer and are not applicable to patients with MBC [6,7]. Thus, effective methods to predict the prognosis of $\mathrm{MBC}$ are required.

The outcome of $\mathrm{MBC}$ patients varies from person to person and was largely correlated with clinical and tumour characteristics of patients [8]. For most of patients with MBC, metastasis disease would progress rapidly involving multiple organ and cause destruction of organ function in a short 
time [3,9]. Meanwhile, a part of MBC patients was reported to achieve tumour remission with systematic therapy and have a relatively prolonged survival [10]. Several populationbased studies have shown that MBC patients with longer metastasis-free interval (MFI $>5$ years) had a superior prognosis to those with a shorter MFI (MFI $<5$ years) [11,12]. In 2015, a study of $850 \mathrm{MBC}$ patients found that those with de novo stage IV BC or with an MFI $>24$ months had a better survival than those with an MFI $<24$ months [13]. In addition, prognosis of patients with different location and quantity of metastatic lesions varies a lot. The spread of metastatic disease to the viscera or the central nervous system (CNS) is usually rapid and causes severe complications and organ failure, which leads to death; in contrast, metastases to the bones or soft tissues could be controlled for a long period by multidisciplinary therapy [14]. Furthermore, patients with primarily metastatic involvement at a single site have better outcomes and are more likely to have improved survival after local surgery than those with metastatic lesions at multiple sites [15]. Hormone receptor (HR) status is an important prognostic factor in breast cancer [16]. HR-positive MBC shows a favourable response to endocrine therapy, which largely prolongs the survival of MBC patients [17]. Triplenegative breast cancer cells, which are negative for HRs, tend to disseminate much earlier and progress more rapidly than cancer cells expressing HRs [18]. Although there have been many reports regarding the MFI, metastasis organ, or HR status to the prognosis of the $\mathrm{MBC}$, there remained no model incorporating these indexes to estimate the survival probability of the MBC.

A nomogram is a graphical calculating model constructed with known prognostic factors that generates a numerical probability of a clinical event [19]. In breast cancer, nomograms have been widely used as a prediction model to predict specific outcomes (such as disease recurrence and distant metastasis) $[20,21]$. Our study aimed to study trend of mortality risk in $\mathrm{MBC}$ and construct a nomogram to quantifying the survival probability of breast cancer patients with distant metastasis.

\section{Materials and Methods}

\section{Study population}

We retrospectively reviewed the medical data of patients who were diagnosed with MBC at Sun Yat-sen University Cancer Center between January 2004 and July 2013. The inclusion criteria were as follows: (1) pathological diagnosis of breast cancer; (2) diagnosis of MBC on imaging or biopsy examination; and (3) age less than 70 years. Patients were excluded if any of the following applied: (1) unknown date of initial breast cancer diagnosis, (2) unclear date of MBC diagnosis, (3) no follow-up information, or (4) history of other cancer or presence of synchronous malignant tumours.

\section{Clinical data collection}

The clinical and histological characteristics of the patients were obtained from the database of Sun Yat-sen University Cancer Center. Metastatic disease was diagnosed using pathological biopsy or imaging examinations, such as ultrasound / contrast-enhanced ultrasound, computed tomography scan, magnetic resonance imaging, positron emission tomography-computed tomography, and bone scan. The clinical stages were stratified based on the eighth American Joint Committee on Cancer/TNM staging system [6]. Human epidermal growth factor receptor 2 (HER2) amplification was defined as " $3+$ " staining on immunohistochemistry or a positive result on fluorescence in situ hybridisation (FISH), while no-amplification was defined as " $1+$ " or "- staining on immunohistochemistry or negative result on FISH.

Overall survival (OS) was defined as the interval between the date of diagnosis of MBC and the date of death. Patient age was recorded at the date of initial diagnosis of breast cancer. The MFI was calculated from the date of diagnosis of breast cancer to the date of detection of MBC. We divided patients into five groups based on MFI: (1) primary stage IV disease, (2) MFI $<6$ months, (3) MFI of 6 months- 2 years, (4) MFI of 2-5 years, and (5) MFI $>5$ years [22]. The number of metastasis sites, as observed on imaging examination, was stratified as 1 and $>1$. The location of metastasis sites was categorized as follows: visceral/CNS metastasis, bone or soft-tissue (distant lymph nodes, skin, and subcutaneous tissues) metastasis, and multiple metastases (patients with both visceral/CNS and bone/soft-tissue metastasis). Tumour mass was classified as $\leq 2 \mathrm{~cm},>2$ and $\leq 5 \mathrm{~cm}$, and $>5 \mathrm{~cm}$ [23]. Patients with locoregional lymph node metastasis were considered to have a positive lymph node status [19].

\section{Nomogram development and statistical analysis}

We used the chi-square and Fisher exact test to compare the clinical and pathological characteristics between the training and validation cohorts. Survival probability and case fatality rate (CFR) in entire group were assessed by the Kaplan-Meier method. CFR was defined as the risk of death from MBC per 6 months. We identified the year when significant changes of CFR took place by Joinpoint regression analysis [24]. Six hundred thirty-four patients diagnosed with MBC between January 2004 and July 2011 were divided 
Table 1. Characteristics of patients with metastatic breast cancer

\begin{tabular}{|c|c|c|c|}
\hline Variable & $\begin{array}{l}\text { Training cohort } \\
\qquad(\mathrm{n}=634)\end{array}$ & $\begin{array}{l}\text { Validation cohort } \\
\qquad(n=159)\end{array}$ & p-value \\
\hline \multicolumn{4}{|l|}{ Age at diagnosis (yr) } \\
\hline$<35$ & $132(20.8)$ & $25(15.7)$ & 0.127 \\
\hline $35-50$ & $300(47.3)$ & $71(44.7)$ & \\
\hline$>50$ & $202(31.9)$ & $63(39.6)$ & \\
\hline \multicolumn{4}{|l|}{ Menopause status } \\
\hline Premenopausal & $434(68.5)$ & $99(62.7)$ & 0.185 \\
\hline Menopausal & $200(31.5)$ & $60(37.3)$ & \\
\hline \multicolumn{4}{|l|}{ Tumour size $(\mathrm{cm})$} \\
\hline$\leq 2$ & $128(20.2)$ & $27(17.0)$ & 0.373 \\
\hline$>2$ and $\leq 5$ & $261(41.2)$ & $78(49.1)$ & \\
\hline$>5$ & $147(23.2)$ & $41(25.8)$ & \\
\hline Unknown & $98(15.5)$ & $13(8.2)$ & \\
\hline \multicolumn{4}{|c|}{ Axillary lymph node involvement } \\
\hline Yes & $483(76.2)$ & $123(77.4)$ & 0.242 \\
\hline No & 139 (21.9) & $36(22.6)$ & \\
\hline Unknown & $12(1.9)$ & 0 & \\
\hline \multicolumn{4}{|l|}{ MFI } \\
\hline Primary stage IV & $194(30.6)$ & $64(40.3)$ & $<0.001$ \\
\hline$<6 \mathrm{mo}$ & $57(9.0)$ & $17(10.7)$ & \\
\hline $6 \mathrm{mo}-2 \mathrm{yr}$ & $203(32.0)$ & $23(14.5)$ & \\
\hline $2-5 \mathrm{yr}$ & $146(23.0)$ & $50(31.4)$ & \\
\hline$>5 \mathrm{yr}$ & $34(5.4)$ & $5(3.1)$ & \\
\hline \multicolumn{4}{|l|}{ No. of metastases } \\
\hline 1 & $382(60.3)$ & $84(52.8)$ & 0.105 \\
\hline$>1$ & $252(39.7)$ & $75(47.2)$ & \\
\hline \multicolumn{4}{|l|}{ Metastasis sites } \\
\hline Visceral or CNS & $191(30.1)$ & $38(23.9)$ & 0.243 \\
\hline Bone or soft tissue & $234(36.9)$ & $60(37.7)$ & \\
\hline Multiple & $209(33.0)$ & $61(38.4)$ & \\
\hline \multicolumn{4}{|c|}{ Hormone receptor status } \\
\hline $\mathrm{HR}+$ & $432(68.1)$ & $107(67.3)$ & 0.850 \\
\hline HR- & $202(31.9)$ & $52(32.7)$ & \\
\hline \multicolumn{4}{|l|}{ HER2 receptor status } \\
\hline Amplified & $213(33.6)$ & $57(35.8)$ & 0.261 \\
\hline Non-amplified & $369(58.2)$ & $95(59.7)$ & \\
\hline Equivocal $(2+)$ & $52(8.2)$ & $7(4.5)$ & \\
\hline \multicolumn{4}{|l|}{ Type of surgery } \\
\hline MRM & $525(82.8)$ & $120(75.5)$ & 0.103 \\
\hline BCT & $23(3.6)$ & $4(2.5)$ & \\
\hline Tumour resection & $5(0.8)$ & $2(1.2)$ & \\
\hline No & $81(12.8)$ & $33(20.8)$ & \\
\hline \multicolumn{4}{|l|}{ Endocrine therapy } \\
\hline Yes & $381(60.1)$ & $76(47.8)$ & 0.002 \\
\hline No & $194(30.6)$ & $54(34.0)$ & \\
\hline Unknown & $59(9.3)$ & $29(18.2)$ & \\
\hline \multicolumn{4}{|l|}{ Anti-HER2 therapy } \\
\hline Yes & $117(18.5)$ & $31(19.5)$ & 0.978 \\
\hline No & $509(80.3)$ & $125(78.6)$ & \\
\hline Unknown & $8(1.3)$ & $3(1.9)$ & \\
\hline
\end{tabular}

(Continued to the next page) 
Table 1. Continued

\begin{tabular}{|c|c|c|c|}
\hline Variable & $\begin{array}{l}\text { Training cohort } \\
\qquad(n=634)\end{array}$ & $\begin{array}{l}\text { Validation cohort } \\
\qquad(n=159)\end{array}$ & p-value \\
\hline \multicolumn{4}{|c|}{ Chemotherapy (first-line regimen) } \\
\hline Yes & $616(97.2)$ & $151(95.0)$ & 0.082 \\
\hline No & $16(2.5)$ & $5(3.1)$ & \\
\hline Unknown & $2(0.3)$ & $3(1.9)$ & \\
\hline \multicolumn{4}{|l|}{ Radiotherapy } \\
\hline Yes & $328(51.7)$ & $63(60.4)$ & 0.008 \\
\hline No & $305(48.1)$ & $96(39.6)$ & \\
\hline Unknown & $1(0.2)$ & 0 & \\
\hline \multicolumn{4}{|l|}{ Vital status } \\
\hline Alive & $286(45.1)$ & $104(65.4)$ & $<0.001$ \\
\hline Death & $348(54.9)$ & $55(34.6)$ & \\
\hline
\end{tabular}

Values are presented as number (\%). MFI, metastasis-free interval; CNS, central nervous system; HR, hormone receptor; HER2, human epidermal growth factor receptor 2; MRM, modified radical mastectomy; $\mathrm{BCT}$, breast conserving therapy.

into training cohort while $159 \mathrm{MBC}$ cases diagnosed between August 2011 and July 2013 were stratified as internal validation cohort. Univariable and multivariable analysis with Cox hazard regression in the training cohort were used to assess the correlation of survival with clinical and pathological characteristics. A p-value of less than 0.05 was considered significant.

We constructed a nomogram model based on the results of the multivariable analysis with the Cox hazard model. The survival probability of the patients could be assumed by summing the scores of the factors and higher score represent the higher risk of mortality. The effectiveness of the nomogram was tested in regards to discrimination and calibration. The concordance index (c-index) along with the 95\% confidence interval $(95 \% \mathrm{CI})$ of the c-index was used to quantify discrimination, which ranged from 0 to $1(<0.5$, absolute discordance; 0.5 , equal concordance to chance; and 1 , best concordance). We used calibration curves to compare the predicted survival with the observed survival at 1 and 3 years in both the training and validation cohorts. More specific details about nomogram construction have been provided in Supplementary Methods.

\section{Ethical statement}

Ethical approval was obtained from the respective Institutional Review Boards of the Ethics Committee of Sun Yat-sen University Cancer Center (YB2017-034). All patients provided written informed consent to participate in this study.

\section{Results}

\section{Patient characteristics}

This study involved a total of 793 MBC patients. The median follow-up duration and the median age of the patients were 42.5 months and 45 years, respectively. The training cohort contained 634 patients, and the validation cohort contained 159 patients. The median survival since diagnosis of MBC was 40.5 months and 31.1 months in the training and validation cohorts, respectively. Table 1 compares clinical and tumour characteristics between the training and validation cohorts.

Survival analysis of entire group of MBC showed that median OS among patients with primary stage IV disease, MFI $<6$ months, MFI of 6 months- 2 years, MFI of 2-5 years, and MFI > 5 years was 54.1, 53.2, 26.6, 39.3, and 58.8 months, respectively ( $p<0.001$, log-rank test). MBC patients with an MFI of 6 months-2 years had the worst prognosis $(p<0.001$, log-rank test). There were no significant differences in OS among patients with primary stage IV disease, MFI $<6$ months, and MFI $>5$ years $($ MFI $>5$ years vs. MFI $<6$ months, $\mathrm{p}=0.612 ; \mathrm{MFI}>5$ years vs. primary stage IV disease, $\mathrm{p}=0.470$; and $\mathrm{MFI}<6$ months vs. primary stage IV disease, $\mathrm{p}=0.826$, log-rank test) (S1A Fig.). With adjustment of age, menopause status, tumour size, lymph node involvement, MFI, number of metastasis, location of metastasis site, HR status, and HER2 receptor status, MFI was significantly correlated with outcome in $\mathrm{MBC}(\mathrm{MFI}<6$ months vs. primary stage IV disease: HRs, 1.125; $\mathrm{p}=0.607$; MFI of 6 months-2 years vs. primary stage IV disease: HRs, 1.851; $\mathrm{p}<0.001$; MFI 
Table 2. Trend of mortality risk for patients with metastatic breast cancer (Joinpoint model)

\begin{tabular}{|c|c|c|c|c|c|c|c|}
\hline & $\begin{array}{l}\text { Time period } \\
\text { (mo) }\end{array}$ & $\begin{array}{l}\text { No. of } \\
\text { deaths }\end{array}$ & CFR $(\%)$ & SEM & $\begin{array}{c}\text { Cumulate } \\
\text { survival rate }(\%)\end{array}$ & $\mathrm{APC}$ & $95 \% \mathrm{CI}$ \\
\hline \multirow[t]{3}{*}{ Trend 1} & 0 to 6 & 42 & 5.4 & 0.01 & 94.6 & $21.6^{*}$ & 1.0 to 46.3 \\
\hline & 7 to 12 & 68 & 9.6 & 0.01 & 85.5 & & \\
\hline & 13 to 18 & 71 & 11.6 & 0.01 & 75.6 & & \\
\hline \multirow[t]{7}{*}{ Trend 2} & 19 to 24 & 59 & 11.5 & 0.01 & 66.9 & $-4.5^{*}$ & -8.2 to -0.7 \\
\hline & 25 to 30 & 53 & 12.7 & 0.02 & 58.4 & & \\
\hline & 31 to 36 & 26 & 8.3 & 0.02 & 53.5 & & \\
\hline & 37 to 42 & 22 & 8.9 & 0.02 & 48.8 & & \\
\hline & 43 to 48 & 17 & 8.6 & 0.02 & 44.6 & & \\
\hline & 49 to 54 & 9 & 5.8 & 0.02 & 42.0 & & \\
\hline & 55 to 60 & 8 & 6.6 & 0.02 & 39.2 & & \\
\hline
\end{tabular}

CFR, case fatality rate; SEM, standard error of mean; APC, annual percentage change; $\mathrm{CI}$, confidence interval. * $\mathrm{p}<0.05$.

A

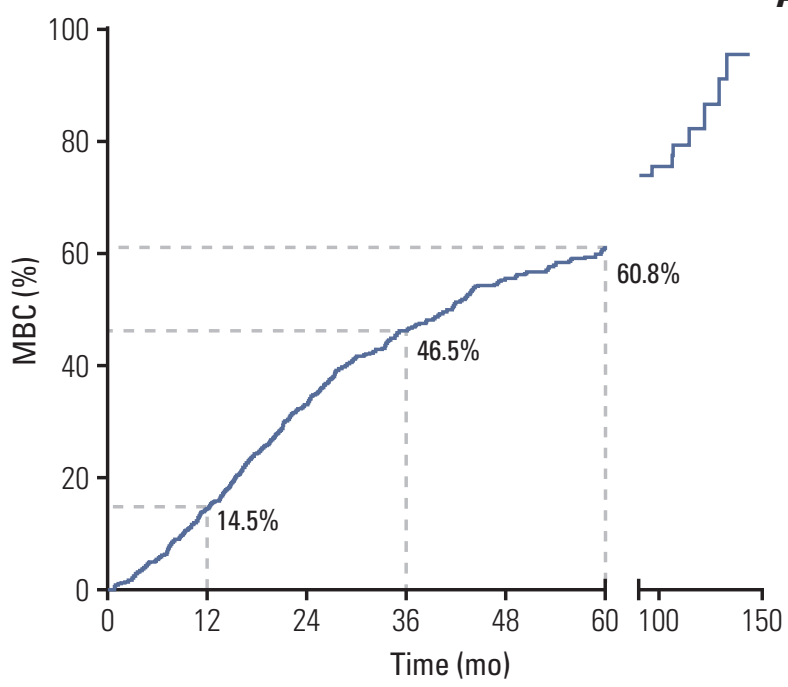

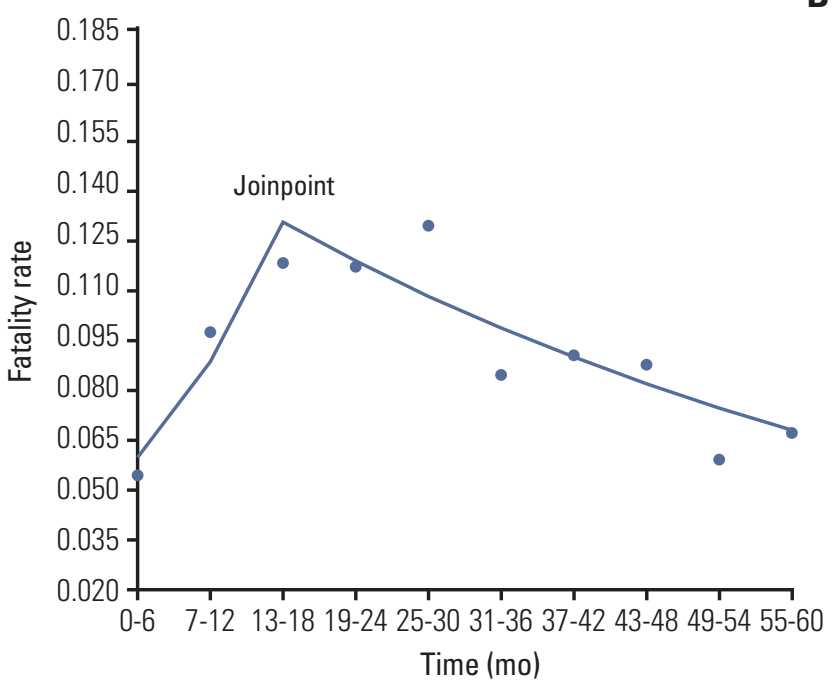

Fig. 1. Mortality risk of metastatic breast cancer (MBC) patients. (A) Kaplan-Meier survival curves estimate case fatality rate of MBC patients: the 1-, 3-, and 5-year fatality rate were 14.5\%, 46.5\%, and 60.8\%, respectively. (B) Analysis of trend of fatality rate per month by Joinpoint regression: the joinpoint of lines was located at 13-18 (95\% confidence interval [CI], [13-18] to [25-30]); trend 1 of fatality rate ranged from 0 to 18th month (annual percentage change [APC], 21.6; 95\% CI, 1.0 to 46.3 ; $\mathrm{p}<0.05$ ); trend 2 of fatality rate ranges from 19th to 60th month (APC, $-4.5 ; 95 \% \mathrm{CI},-8.2$ to $-0.7 ; \mathrm{p}<0.05$ ).

of 2-5 years: HRs, $1.331 ; \mathrm{p}=0.063 ; \mathrm{MFI}>5$ years vs. primary stage IV disease: $\mathrm{HRs}, 0.836 ; \mathrm{p}=0.571$; multivariable analysis) (S2 Table). Correlation between clinical factors (age, metastasis location, and HR status) and OS was calculated by survival curves (S1B-S1D Fig., S2 Table).

\section{Fatality risk}

The 1-, 3-, and 5-year CFR of MBC was $14.5 \%, 46.5 \%$, and $60.8 \%$, respectively (Table 2 , Fig. 1A). Joinpoint model fitted a serials of straight line to the CFR. Line of joinpoint was continuously rising in the first 18 months as the fatality rate was increasing (annual percentage change [APC], 21.6; 95\% CI, 1.0 to $46.3 ; \mathrm{p}<0.05)$. After reached the peak at the joinpoint 
Table 3. Univariable and multivariable Cox regression model in metastatic breast cancer (training cohort)

\begin{tabular}{|c|c|c|c|c|c|c|}
\hline Characteristic & $\begin{array}{l}\text { Univariable } \\
\text { hazard ratios }\end{array}$ & $95 \% \mathrm{CI}$ & p-value & $\begin{array}{l}\text { Multivariable } \\
\text { hazard ratios }\end{array}$ & $95 \% \mathrm{CI}$ & p-value \\
\hline Age (yr) & & & 0.001 & & & 0.005 \\
\hline$<35$ & 1 & & & 1 & & \\
\hline $35-50$ & 0.500 & $0.334-0.749$ & 0.001 & 0.526 & $0.306-0.906$ & 0.020 \\
\hline$>50$ & 0.607 & $0.277-1.328$ & 0.231 & 0.873 & $0.214-1.452$ & 0.374 \\
\hline \multicolumn{7}{|l|}{ Menopause status } \\
\hline Menopause vs. menses & 0.102 & $0.010-1.015$ & 0.052 & & & \\
\hline Tumour size (cm) & & & 0.480 & & & \\
\hline$\leq 2$ & 1 & & & & & \\
\hline$>2$ and $\leq 5$ & 1.266 & $0.802-1.998$ & 0.312 & & & \\
\hline$>5$ & 1.214 & $0.583-2.525$ & 0.604 & & & \\
\hline \multicolumn{7}{|l|}{ Lymph node involvement } \\
\hline Yes vs. no & 1.382 & $0.577-3.310$ & 0.468 & & & \\
\hline MFI & & & 0.006 & & & 0.007 \\
\hline Primary stage IV & 1 & & & 1 & & \\
\hline$<6 \mathrm{mo}$ & 0.872 & $0.512-1.484$ & 0.613 & 0.670 & $0.372-1.209$ & 0.184 \\
\hline $6 \mathrm{mo}-2 \mathrm{yr}$ & 1.257 & $0.752-2.103$ & 0.383 & 0.780 & $0.369-1.648$ & 0.515 \\
\hline $2-5 \mathrm{yr}$ & 0.773 & $0.371-1.612$ & 0.492 & 0.469 & $0.157-1.402$ & 0.176 \\
\hline$>5 \mathrm{yr}$ & 0.468 & $0.170-1.288$ & 0.142 & 0.208 & $0.048-0.905$ & 0.036 \\
\hline \multicolumn{7}{|l|}{ No. of metastases } \\
\hline$>1$ vs. 1 & 0.596 & $0.142-2.502$ & 0.480 & & & \\
\hline Metastasis site & & & 0.001 & & & $<0.001$ \\
\hline Visceral/CNS & 1 & & & 1 & & \\
\hline Bone/Soft tissue & 0.483 & $0.324-0.721$ & $<0.001$ & 0.745 & $0.478-1.163$ & 0.195 \\
\hline Multiple & 0.477 & $0.111-2.054$ & 0.320 & 1.991 & $0.715-5.544$ & 0.187 \\
\hline \multicolumn{7}{|l|}{ HR status } \\
\hline HR+ vs. HR- & 0.321 & $0.131-0.788$ & 0.013 & 0.270 & $0.102-0.711$ & 0.008 \\
\hline HER2 receptor status & & & 0.937 & & & \\
\hline Non-amplification & 1 & & & & & \\
\hline Amplification & 0.838 & $0.320-2.191$ & 0.718 & & & \\
\hline Not known or equivocal $(2+)$ & 0.702 & $0.090-5.456$ & 0.735 & & & \\
\hline
\end{tabular}

CI, confidence interval; MFI, metastasis-free interval; CNS, central nervous system; HR, hormone receptor; HER2, human epidermal growth factor receptor 2 .

(joinpoint, 13 to 18; 95\% CI, [13-18] to [25-30]), the line began to fall indicating the decreasing fatality rate in MBC (APC, $-4.5 ; 95 \%$ CI, -8.2 to $-0.7 ; \mathrm{p}<0.05$ ) (Table 2, Fig. 1B).

\section{Outcome-prediction nomogram in the training cohort}

Of the 634 patients in the training cohort, $348(54.9 \%)$ died of breast cancer during the follow-up period. Univariable analysis using the Cox hazard model identified the following significant factors (Table 3$)$ : age ( $35-50$ years vs. $<35$ years; $>50$ years vs. $<35$ years; $p=0.001)$, MFI $(\mathrm{MFI}<6$ months vs. primary stage IV disease; MFI of 6 months- 2 years vs. primary stage IV disease; MFI of 2-5 years vs. primary stage IV disease; MFI > 5 years vs. primary stage IV disease; $\mathrm{p}=0.006$ ), location of metastasis sites (bone/soft tissue vs. viscera/ CNS; multiple vs. viscera/CNS; $\mathrm{p}=0.001$ ), and HR status (positive vs. negative, $\mathrm{p}=0.013$ ). The multivariable Cox hazard model showed that the following factors were independently correlated with patient survival (Table 3): age (35-50 years vs. $<35$ years; $>50$ years vs. $<35$ years; $p=0.005)$, MFI (MFI $<6$ months vs. primary stage IV disease; MFI of 6 months-2 years vs. primary stage IV disease; MFI of 2-5 years vs. primary stage IV disease; MFI $>5$ years vs. primary stage IV disease; $p=0.007$ ), location of metastasis site (bone/ soft tissue vs. viscera/CNS; multiple vs. viscera/CNS; $\mathrm{p}<$ 0.001 ), and HR status (positive vs. negative; $\mathrm{p}=0.008$ ).

A nomogram based on data from the training cohort was developed for the prediction of OS at 1 and 3 years. We con- 


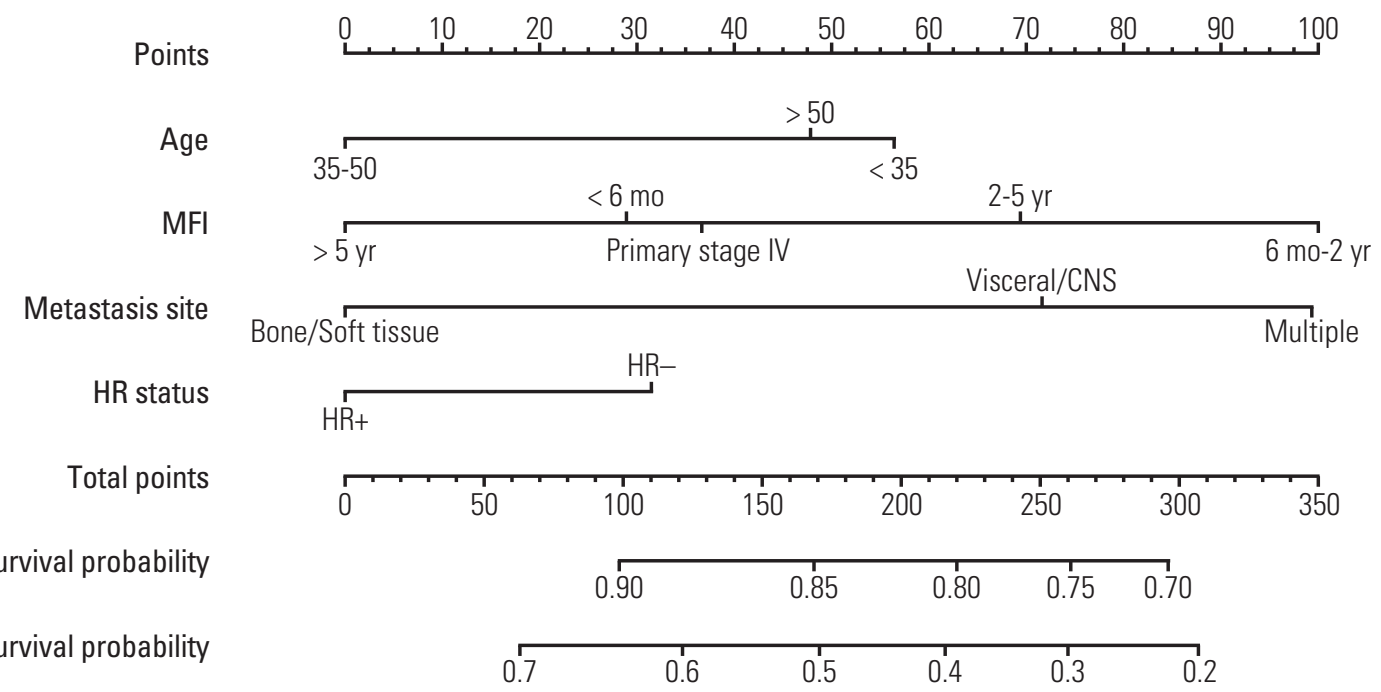

Fig. 2. Prognostic nomogram for metastatic breast cancer patients with factors, including age, metastasis-free interval (MFI), location of metastasis site, and hormone receptor (HR) status. Points are defined based on the prognostic contribution of the factors (top). Points summing the contribution of age, MFI, location of metastasis site, and HR status are translated to the survival probability at 1 and 3 years (bottom). CNS, central nervous system.

A
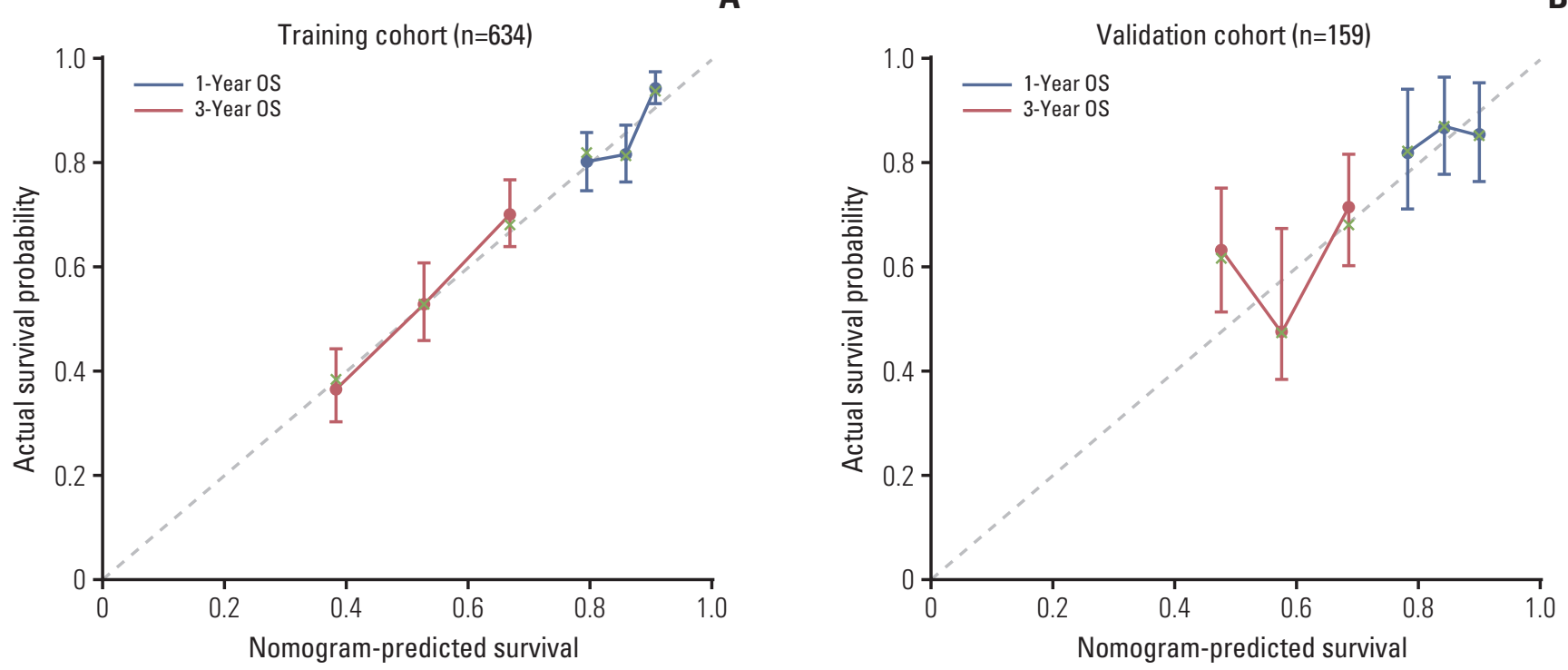

Fig. 3. Calibration plots for predicting patient survival at 1 and 3 years in the training and validation cohorts. Calibration plots of survival probability in training (A) and validation (B) cohort. Probability of survival based on the nomogram has been listed on the x-axis, while actual probability of survival has been listed on the y-axis. OS, overall survival.

structed the nomogram with the following factors: age $(<35$, $35-50$, or $>50$ years), MFI (primary stage IV disease, MFI $<6$ months, MFI 6 months- 2 years, MFI 2-5 years, or MFI $>5$ years), location of metastasis (viscera/CNS, bone/ soft tissue, or multiple), and HR status (positive or negative) (Fig. 2). The score scale of MFI ranged from 0 to 100, and was larger than 
the scales for the other factors (Fig. 3). The score for an MFI of 6 months-2 years was much higher than that for primary stage IV disease, MFI $<6$ months, MFI of $2-5$ years, and MFI $>5$ years. The score for MFI $>5$ years was ranked for 0 point while MFI 6 months-2 years was ranked for 100 point. The c-index of this model was 0.69 (95\% CI, 0.62 to 0.71$)$.

\section{Validation of the nomogram}

In all, 159 MBC patients diagnosed between August 2011 and July 2013 were stratified as internal validation cohort. As shown in Table 1, there was no significant difference between the validation and training cohorts with regard to clinical and tumour characteristics. In the validation cohort, $55 \mathrm{MBC}$ patients $(34.6 \%)$ died of breast cancer during the study period. The c-index of the nomogram in the external validation cohort was 0.67 (95\% CI, 0.63 to 0.71$)$. The calibration curve indicated good consistency at 1 and 3 years between the training and validation cohorts (Fig. 3).

\section{Discussion}

This is the first study to develop and validate a nomogram which could calculate the 1- and 3-year survival probability of MBC. We have shown that the 1-, 3-, and 5-year CFR of $\mathrm{MBC}$ was $14.5 \%, 46.5 \%$ and $60.8 \%$, and mortality risk of MBC was increasing and reached the summit between the 18th and 30th month after the detection of MBC.

MBC remains an incurable disease wit poor prognosis among breast cancer [25]. Our study showed that almost half of MBC patients (46.5\%) would die of cancer within 3 years, which was concordant with the previuos report [10]. The purpose of treatment for MBC is to reduce tumour burden, relieve symptoms, and improve the quality of life [3]. Although many therapeutic approaches have been developed in $\mathrm{MBC}$, the selection of an optimal regimen remains a great challenge in clinical practice [26]. Patients with stable disease and low tumour burden might benefit from aggressive multidisciplinary therapy by delaying tumour progression and prolonging survival. However, in most MBC patients, diseases progress rapidly which would soon destruct organ-function and cause death, and application of aggressive therapy in such MBC patients might increase side effects without improving outcomes. In our study, mortality risk of $\mathrm{MBC}$ was increasing and reached the summit soon (13th to 30th month) after the detection of MBC. Consistently, survival analysis showed that almost half of patients died within 3 years since diagnosis of MBC. These results indicated that majority of $\mathrm{MBC}$ patients were unresponsive to alleviative treatment or became resistent to systematic therapy in a short period, which makes aggressive multidisciplinary therapy useless. Thus, methods to predict which patients may be at a low risk of mortality and suitable to aggressive therapy are required for the individualized treatment of MBC patients. Interestingly, our model was an effective statistical method to predict mortality risk in MBC patients. Patients with MFI $>5$ years, HR-positive status, young age, or bone/soft-tissue metastases tended to have better outcomes. Physicians may be able to quantify the mortality risk of $\mathrm{MBC}$ patients and individualize treatments based on our nomogram.

Cancer researchers are increasingly interested in statistical methods of survival or recurrence prediction in breast cancer. Although several studies have reported that short MFI, HRnegative tumour, and viscera metastasis are correlated with worse outcomes in $\mathrm{MBC}$, models combining clinical and tumour characteristics might be more effective to predict the outcomes of MBC patients [27]. Nomograms based on the Cox regression model have been proved to be superior to other statistical models (such as probability tables, regression tree analyses, and risk groupings) $[19,28]$. We constructed a nomogram combining age, MFI, location of metastasis site, and HR status. In this model, MFI played a major role in the scoring system. An MFI > 5 years yielded the lowest score, while an MFI of 6 months- 2 years was associated with a higher score than those for primary stage IV disease, MFI of 2-5 years, and MFI $\leq 6$ months. These are consistent with our results that MBC patients with an MFI of 6 months- 2 years had the worst prognosis, while those with an MFI $>5$ years had the longest survival. The poor survival in the group with an MFI of 6 months-2 years might be attributable to tumour resistance or unresponsiveness to systemic therapy. Previously, study has suggested that patients with triple-negative breast cancer, which lacks therapeutic targets and is associated with poor outcomes, tended to have a much higher risk of disease recurrence than did patients with other types of breast cancer [18]. HR-positive breast cancer, which can be effectively treated with current therapeutic methods and is associated with good outcomes, tends to develop distant metastases beyond 5 years after the diagnosis $[4,29]$. This might partly explain the better survival of patients with an MFI $>5$ years than that of patients with an MFI of 6 months2 years. One study has reported that women with primary stage IV breast cancer had superior survival to that of relapsed-MBC patients, as the former were chemotherapynaïve $[13,22]$. In our study, the survival curves showed that patients with primary stage IV disease had a similar prognosis to that of patients with an MFI > 5 years. Interestingly, our study also showed that the prognosis of patients with an MFI $<6$ months was equal to that of patients with an MFI $>5$ years or those with primary stage IV disease. We 
assumed that a portion of patients with an MFI $<6$ months might have had occult metastatic lesions long before they were detected, and could therefore be considered to have a low tumour burden.

At last, we evaluated the effectiveness of the nomogram via discrimination calibration and the c-index. In our study, the c-index was good in both the training and validation cohorts, and the calibration curves of both groups indicated a favourable prediction stability of our model.

The current study has several limitations. First, this was a single-centre study that the validation cohort came from the same cohort with training set, and the internal validity may give over-optimistic result. However, we have included 793 metastasis breast cancer patients with complete clinical and follow-up information, which may limit the number of the cohort but guaranteed the accuracy of the model. Second, gene signatures were unavailabe in our study population. As many studies have revealed that tumour biology and patient outcomes are significantly correlated with gene expression, gene signatures should be incorporated into the nomogram $[9,30]$. Finally, our study is retrospective in nature; we will carry out a prospective study to evaluate our nomogram in the future.

In conclusion, we have constructed a nomogram including MFI, location of metastasis site, age, and HR status, which is an effective model to predict survival in MBC patients. Our model could help identify patients with long life expectancies and thus guide clinical decision-making.

\section{Electronic Supplementary Material}

Supplementary materials are available at Cancer Research and Treatment website (http:// www.e-crt.org).

\section{Conflicts of Interest}

Conflict of interest relevant to this article was not reported.

\section{References}

1. DeSantis C, Ma J, Bryan L, Jemal A. Breast cancer statistics, 2013. CA Cancer J Clin. 2014;64:52-62.

2. Miao H, Hartman M, Bhoo-Pathy N, Lee SC, Taib NA, Tan EY, et al. Predicting survival of de novo metastatic breast cancer in Asian women: systematic review and validation study. PLoS One. 2014;9:e93755.

3. Cardoso F, Costa A, Senkus E, Aapro M, Andre F, Barrios CH, et al. 3rd ESO-ESMO International Consensus Guidelines for Advanced Breast Cancer (ABC 3). Ann Oncol. 2017;28:16-33.

4. Turner NC, Neven P, Loibl S, Andre F. Advances in the treatment of advanced oestrogen-receptor-positive breast cancer. Lancet. 2017;389:2403-14.

5. Harbeck N, Gnant M. Breast cancer. Lancet. 2017;389:1134-50. 6. Amin MB, Greene FL, Edge SB, Compton CC, Gershenwald JE, Brookland RK, et al. The eighth edition AJCC cancer staging manual: continuing to build a bridge from a populationbased to a more "personalized" approach to cancer staging. CA Cancer J Clin. 2017;67:93-9.

7. MammaPrint reduces breast cancer overtreatment. Cancer Discov. 2016;6:OF4.

8. Kennecke H, Yerushalmi R, Woods R, Cheang MC, Voduc D, Speers $\mathrm{CH}$, et al. Metastatic behavior of breast cancer subtypes. J Clin Oncol. 2010;28:3271-7.

9. Lin C, Liao W, Jian Y, Peng Y, Zhang X, Ye L, et al. CGI-99 promotes breast cancer metastasis via autocrine interleukin- 6 signaling. Oncogene. 2017;36:3695-705.

10. Lee ES, Jung SY, Kim JY, Kim JJ, Yoo TK, Kim YG, et al. Identifying the potential long-term survivors among breast cancer patients with distant metastasis. Ann Oncol. 2016;27:828-33.
11. den Brok WD, Speers CH, Gondara L, Baxter E, Tyldesley SK, Lohrisch CA. Survival with metastatic breast cancer based on initial presentation, de novo versus relapsed. Breast Cancer Res Treat. 2017;161:549-56.

12. Eng LG, Dawood S, Sopik V, Haaland B, Tan PS, Bhoo-Pathy $\mathrm{N}$, et al. Ten-year survival in women with primary stage IV breast cancer. Breast Cancer Res Treat. 2016;160:145-52.

13. Lobbezoo DJ, van Kampen RJ, Voogd AC, Dercksen MW, van den Berkmortel F, Smilde TJ, et al. Prognosis of metastatic breast cancer: are there differences between patients with de novo and recurrent metastatic breast cancer? Br J Cancer. 2015;112:1445-51.

14. Wang H, Zhang C, Zhang J, Kong L, Zhu H, Yu J. The prognosis analysis of different metastasis pattern in patients with different breast cancer subtypes: a SEER based study. Oncotarget. 2017;8:26368-79.

15. Rapiti E, Verkooijen HM, Vlastos G, Fioretta G, Neyroud-Caspar I, Sappino AP, et al. Complete excision of primary breast tumor improves survival of patients with metastatic breast cancer at diagnosis. J Clin Oncol. 2006;24:2743-9.

16. Gong Y, Liu YR, Ji P, Hu X, Shao ZM. Impact of molecular subtypes on metastatic breast cancer patients: a SEER populationbased study. Sci Rep. 2017;7:45411.

17. Robertson JF, Bondarenko IM, Trishkina E, Dvorkin M, Panasci L, Manikhas A, et al. Fulvestrant 500 mg versus anastrozole $1 \mathrm{mg}$ for hormone receptor-positive advanced breast cancer (FALCON): an international, randomised, doubleblind, phase 3 trial. Lancet. 2016;388:2997-3005.

18. Denkert C, Liedtke C, Tutt A, von Minckwitz G. Molecular 
alterations in triple-negative breast cancer: the road to new treatment strategies. Lancet. 2017;389:2430-42.

19. Graesslin O, Abdulkarim BS, Coutant C, Huguet F, Gabos Z, Hsu L, et al. Nomogram to predict subsequent brain metastasis in patients with metastatic breast cancer. J Clin Oncol. 2010;28:2032-7.

20. Lim YJ, Lee SW, Choi N, Kwon J, Eom KY, Kang E, et al. A novel prognostic nomogram for predicting risks of distant failure in patients with invasive breast cancer following postoperative adjuvant radiotherapy. Cancer Res Treat. 2018;50: $1140-8$

21. Kim MK, Han W, Moon HG, Ahn SK, Kim J, Lee JW, et al. Nomogram for predicting breast conservation after neoadjuvant chemotherapy. Cancer Res Treat. 2015;47:197-207.

22. Dawood S, Broglio K, Ensor J, Hortobagyi GN, Giordano SH. Survival differences among women with de novo stage IV and relapsed breast cancer. Ann Oncol. 2010;21:2169-74.

23. Thomas A, Khan SA, Chrischilles EA, Schroeder MC. Initial surgery and survival in stage IV breast cancer in the United States, 1988-2011. JAMA Surg. 2016;151:424-31.

24. Joinpoint Regression Program, version 4.5.0.1 [Internet]. Bethesda, MD: Statistical Research and Applications Branch, National Cancer Institute; 2017 [cited 2017 Jun 12]. Available from: https://surveillance.cancer.gov/joinpoint/ download.
25. Di Meglio A, Freedman RA, Lin NU, Barry WT, Metzger-Filho $\mathrm{O}$, Keating NL, et al. Time trends in incidence rates and survival of newly diagnosed stage IV breast cancer by tumor histology: a population-based analysis. Breast Cancer Res Treat. 2016;157:587-96.

26. Di Lascio S, Pagani O. Oligometastatic breast cancer: a shift from palliative to potentially curative treatment? Breast Care (Basel). 2014;9:7-14.

27. Park S, Han W, Kim J, Kim MK, Lee E, Yoo TK, et al. Risk factors associated with distant metastasis and survival outcomes in breast cancer patients with locoregional recurrence. J Breast Cancer. 2015;18:160-6.

28. Iasonos A, Schrag D, Raj GV, Panageas KS. How to build and interpret a nomogram for cancer prognosis. J Clin Oncol. 2008;26:1364-70.

29. Hart CD, Migliaccio I, Malorni L, Guarducci C, Biganzoli L, Di Leo A. Challenges in the management of advanced, ER-positive, HER2-negative breast cancer. Nat Rev Clin Oncol. 2015;12:541-52.

30. Wang X, Lin C, Zhao X, Liu A, Zhu J, Li X, et al. Acylglycerol kinase promotes cell proliferation and tumorigenicity in breast cancer via suppression of the FOXO1 transcription factor. Mol Cancer. 2014;13:106. 